\title{
Enhanced detection of Legionnaires' disease by PCR testing of induced sputum and throat swabs
}

\author{
To the Editor:
}

Legionnaires' disease, particularly that caused by non-pneumophila species, is notoriously underdiagnosed $[1,2]$. We recently found a four-fold increase in case detection of Legionnaires' disease through a laboratory-initiated strategy of systematic PCR testing for Legionella species of all lower respiratory specimens from patients with pneumonia or immune compromised status [3]. This strategy relies on the availability of lower respiratory specimens and the recording of relevant clinical information on laboratory requisition forms by clinicians. We recognised that this strategy will miss testing patients who could not expectorate sputum and when inadequate clinical information is written on laboratory requisition forms. To address this diagnostic gap we enhanced case detection by actively identifying patients with communityacquired pneumonia (CAP) and by collecting induced sputum from those unable to expectorate voluntarily. In addition, we evaluated throat swabs as an alternative specimen for PCR testing.

From October 2012 to March 2013 patients admitted to Christchurch Hospital and The Princess Margaret Hospital (both in Christchurch, New Zealand) with CAP and aged $\geqslant 18$ years were recruited. For logistical reasons, recruitment occurred on weekdays only. The study period was chosen to coincide with peak Legionnaires' disease activity in Christchurch [3]. Patients were excluded if the pneumonia was hospital acquired or associated with bronchial obstruction, bronchiectasis or tuberculosis. Patients were not eligible for sputum induction if they required high-flow oxygen or assisted ventilation at enrolment.

Ethical approval was obtained from the New Zealand Northern A Ethics Committee. Informed consent was obtained from the patient or their next of kin with separate informed consent for induced sputum collection.

Legionnaires' disease was defined by a positive sputum PCR, sputum culture or urinary antigen test result in a patient with pneumonia. CAP was defined as an acute illness with clinical features of pneumonia and radiographic pulmonary shadowing that is at least segmental or present in one lobe and is neither preexisting nor because of some other known cause.

Sputum induction was performed on the day it was requested, using ultrasonically nebulised 7\% hypertonic saline for 20 min via a DeVilbiss Ultraneb (DeVilbiss Healthcare, Somerset, PA, USA). Throat swabs were collected using nylon flocked swabs (Floqswabs; Copan flock technologies, Brescia, Italy) and placed in universal transport media. Sputum and throat swabs were tested for Legionella species using PCR. The isolation of DNA from clinical specimens was performed using the SPRI-TE (Beckman Coulter, Auckland, New Zealand) nucleic acid extractor and genomic DNA Extraction Kit as recommended by the manufacturer. Legionella DNA was detected using real-time PCR [2], confirmation of the PCR products that did not have melting curve data consistent with Legionella pneumophila was performed using a realtime PCR with a different gene target [3]. This confirmatory PCR had both genus and Legionella longbeachae specific probes. PCR inhibitor controls were used to validate negative PCR results [3-5]. Urine samples were tested for L. pneumophila serogroup 1 and Streptococcus pneumoniae using the BinaxNOW Legionella Urinary Antigen Card and BinaxNOW S. pneumoniae Antigen Card (Alere, Scarborough, ME, USA), respectively. Blood cultures were taken at the request of attending physicians.

Over the study period, 145 patients were eligible with 114 enrolled. 26 patients did not consent and four who were initially enrolled were excluded when the diagnosis of malignancy $(n=1)$, tuberculosis $(n=2)$ and hospital-acquired pneumonia $(n=1)$ was made. One patient was excluded because pneumonia was not the primary reason for admission.

The median (interquartile range) age of those included was 69 (18-101) years, with 67 (59\%) females. There were $22(19 \%)$ cases of Legionnaires' disease: 16 cases caused by L. longbeachae, three caused by L. pneumophila and three non-typable Legionella species. Of these, 21 were detected by sputum PCR, eight had positive sputum cultures (all PCR positive), and three had positive urinary antigen tests (two were also detected with sputum). The three non-typable Legionella species were all culture negative, urinary antigen 


$\begin{array}{lc}\text { Expectorated sputum } & 13 / 68 \\ \text { Expectorated sputum obtained with nurse or } & 4 / 10 \\ \quad \text { physiotherapist assistance } & \\ \text { Induced sputum } & 4 / 19 \\ \text { Throat swab } & 3 / 114\end{array}$

Data are presented as $\mathrm{n} / \mathrm{N}$.

negative and of insufficient copy number to type. Of the 114 patients, $17(15 \%)$ had positive urinary antigen tests for S. pneumoniae and one (1\%) patient had a positive blood culture for S. pneumoniae.

A summary of the yield of diagnostic specimens is presented in table 1 . Of the 114 cases, 46 (40\%) were unable to spontaneously expectorate sputum. Induced sputum was requested for 31 patients and 15 did not give consent or were ineligible. Of the 31 who consented to have sputum induced, $10(34 \%)$ produced an expectorated sputum sample with nursing or physiotherapist assistance (four were positive for Legionella), 19 underwent sputum induction and two were discharged prior to the procedure. Of the 19 who underwent sputum induction, all produced sputum without adverse events and four tested positive for Legionella. Therefore, eight (36\%) out of 22 cases of Legionnaires' disease would not have been detected without active specimen collection. Of the PCR-positive sputum specimens, $11(52 \%)$ out of 21 had $>10$ squamous epithelial cells per low power field and $12(57 \%)$ out of 21 had $<25$ leukocytes per low power field; criteria often used to indicate poor quality specimens.

Throat swabs were performed on all 114 patients and were positive in only three (3\%), all of whom also had positive sputum specimens.

The identification of Legionella spp. altered the treatment in $16(73 \%)$ patients. In five patients this was due to the inclusion of an agent active against Legionella and in 11 patients there was a rationalisation of antimicrobials.

We found that active specimen collection increased the case detection of Legionnaires' disease in our region by at least one-third over and above PCR testing of routine specimens. During spring and summer in our region, Legionnaires' disease accounted for approximately one-fifth of all cases of adult CAP admitted to hospital, and was more common than confirmed pneumococcal pneumonia.

The diagnostic performance of Legionella PCR has been reported previously but, in brief, it is highly sensitive and specific, with a rapid turn-around time and disease severity correlates with bacterial load $[3,6]$.

Sputum is the preferred diagnostic sample for Legionella PCR testing [2]. However, $>40 \%$ of pneumonia patients in our study were unable to spontaneously expectorate sputum. We managed to obtain a sputum sample through extra assistance or induction in only approximately two-thirds of these cases but, in doing so, still increased the case detection of Legionnaires' disease by $36 \%$. The most common reason for not obtaining sputum was ineligibility for sputum induction. We were cautious in selecting patients for sputum induction to avoid adverse reactions and it was universally well-tolerated. The technique of induced sputum has been shown to be safe by others and could be more widely applied [7].

It has been reported that throat swabs may be useful for diagnosing the cause of lower respiratory tract infections by PCR [8-10]. In our study, a throat swab was positive in a minority of cases and offered no advantage over sputum samples.

The relatively small sample size and study period over only one spring/summer are important limitations of our study. Additionally, the conclusions are only valid in regions with a high incidence of non-pneumophila Legionnaires' disease such as Australasia.

In conclusion, active collection of sputum samples for PCR testing, including by induction, in patients with CAP enhances the case detection of Legionnaires' disease, and is recommended in high-prevalence regions. Throat swabs offer no advantage over sputum samples, but further work is needed to assess their potential role in situations when lower respiratory samples cannot be obtained.

Testing induced sputum samples from those who cannot produce sputum increases diagnosis of Legionnaires' disease http://ow.ly/rm9WD 
Michael J. Maze ${ }^{1,2}$, Sandy Slow ${ }^{3}$, Anne-Marie Cumins ${ }^{4}$, Kenjin Boon ${ }^{4}$, Patricia Goulter ${ }^{5}$, Roslyn G. Podmore ${ }^{6}$, Trevor P. Anderson ${ }^{6}$, Kevin Barratt ${ }^{6}$, Sheryl A. Young ${ }^{6}$, Alan D. Pithie ${ }^{1,4}$, Michael J. Epton ${ }^{2,7}$, Anja M. Werno ${ }^{6}$, Stephen T. Chambers ${ }^{1,3}$ and David R. Murdoch ${ }^{3,6}$

${ }^{1}$ Dept of Infectious Diseases, Christchurch Hospital, Christchurch, ${ }^{2}$ Dept of Respiratory Medicine, Christchurch Hospital, Christchurch, ${ }^{3}$ Dept of Pathology, University of Otago, Christchurch, ${ }^{4}$ Dept of General Medicine, Christchurch Hospital, Christchurch, ${ }^{5}$ Physiotherapy Dept, Christchurch Hospital, Christchurch, ${ }^{6}$ Microbiology Unit, Canterbury Health Laboratories, Christchurch, and ${ }^{7}$ Canterbury Respiratory Research Group, Christchurch, New Zealand.

Correspondence: M.J. Maze, Dept of Respiratory Medicine, Private Bag 4710, Christchurch, New Zealand. E-mail: Michael.Maze@cdhb.health.nz

Received: Nov 042013 | Accepted: Nov 142013 | First published online: Dec 52013

Conflict of interest: None declared.

Acknowledgements: We thank the staff of the General Medicine Service and the Physiotherapy Department of Christchurch Hospital, and of the Microbiology Unit of Canterbury Health Laboratories (both Christchurch, New Zealand) for their invaluable support throughout the project.

\section{References}

Diederen BM. Legionella spp. and Legionnaires' disease. J Infect 2008; 56: 1-12.

Murdoch DR. Diagnosis of Legionella infection. Clin Infect Dis 2003; 36: 64-69.

Murdoch DR, Podmore RG, Anderson TP, et al. Impact of routine systematic PCR testing on case finding for Legionnaires' disease: a pre-post comparison study. Clin Infect 2013; 57: 1275-1281.

4 Reischl U, Linde H-J, Lehn N, et al. Direct detection and differentiation of Legionella spp. and Legionella pneumophila in clinical specimens by dual-colour real-time PCR and melting curve analysis. J Clin Microbiol 2002; 40: 3814-3817.

5 Yang G, Benson R, Pelish T, et al. Dual detection of Legionella pneumophila and Legionella species by real-time PCR targeting the 23S-5S rRNA gene spacer region. Clin Microbiol Infect 2010; 16: 255-261.

6 Diederen BM, Kluytmans AJW, Vandenbroucke-Grauls CM, et al. Utility of real-time PCR for diagnosis of Legionnaires' disease in routine clinical practice. J Clin Microbiol 2008; 46: 671-677.

$7 \quad$ Pizzichini E, Pizzichini MMM, Leigh R, et al. Safety of sputum induction. Eur Respir J 2002; 20: Suppl. 37, 9s-18s.

8 Diederen BM, Peeters MF. Are oropharyngeal swabs suitable as samples for Legionella-specific PCR testing? J Clin Microbiol 2007; 45: 3482-3483.

9 McDonough EA, Metzgar D, Hansen CJ, et al. A cluster of Legionella-associated pneumonia cases in a population of military recruits. J Clin Microbiol 2007; 45: 2075-2077.

10 Ramirez JA, Ahkee S, Tolentino A, et al. Diagnosis of Legionella pneumophila, Mycoplasma pneumoniae, or Chlamydia pneumoniae lower respiratory infection using the polymerase chain reaction on a single throat swab specimen. Diagn Microbiol Infect Dis 1996; 24: 7-14. 